\title{
Progerin elicits disease phenotypes of progeria in mice whether or not it is farnesylated
}

\author{
Shao H. Yang, ${ }^{1}$ Douglas A. Andres, ${ }^{2}$ H. Peter Spielmann, ${ }^{2}$ Stephen G. Young,,$^{1,3}$ and Loren G. Fong \\ 1Department of Medicine, UCLA David Geffen School of Medicine, Los Angeles, California, USA. '2Department of Molecular and Cellular Biochemistry, \\ University of Kentucky, Lexington, Kentucky, USA. ${ }^{3}$ Department of Human Genetics, UCLA David Geffen School of Medicine, Los Angeles, California, USA.
}

\begin{abstract}
Hutchinson-Gilford progeria syndrome (HGPS), a rare disease that results in what appears to be premature aging, is caused by the production of a mutant form of prelamin A known as progerin. Progerin retains a farnesyl lipid anchor at its carboxyl terminus, a modification that is thought to be important in disease pathogenesis. Inhibition of protein farnesylation improves the hallmark nuclear shape abnormalities in HGPS cells and

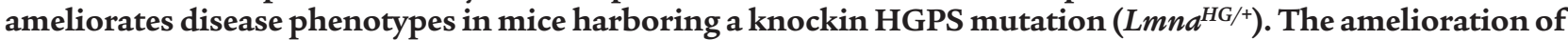
disease, however, is incomplete, leading us to hypothesize that nonfarnesylated progerin also might be capable of eliciting disease. To test this hypothesis, we created knockin mice expressing nonfarnesylated progerin $\left(L m n a^{n H G /+}\right) . L m n a^{n H G /+}$ mice developed the same disease phenotypes observed in $L m n a^{H G /+}$ mice, although the phenotypes were milder, and mouse embryonic fibroblasts (MEFs) derived from these mice contained fewer misshapen nuclei. The steady-state levels of progerin in $L m n a^{n H G /+}$ MEFs and tissues were lower, suggesting a possible explanation for the milder phenotypes. These data support the concept that inhibition of protein farnesylation in progeria could be therapeutically useful but also suggest that this approach may be limited, as progerin elicits disease phenotypes whether or not it is farnesylated.
\end{abstract}

\section{Introduction}

Hutchinson-Gilford progeria syndrome (HGPS) is a rare pediatric progeroid syndrome associated with the production of a mutant form of prelamin A (1-5). Affected children appear normal at birth but soon develop multiple disease phenotypes resembling premature aging, including slow growth, loss of adipose tissue, alopecia, osteoporosis, and occlusive vascular disease (1, 3, 6). HGPS is caused by a $L M N A$ point mutation that alters mRNA splicing and leads to the production of a mutant prelamin $\mathrm{A}$, progerin, that contains a 50-amino acid internal deletion within the carboxyl-terminal domain of the protein $(1,7)$. Progerin, like wild-type prelamin A (8), contains a carboxyl-terminal CaaX motif, which triggers protein farnesylation. The farnesyl lipid is added to the thiol group of the cysteine within the CaaX motif by a cytosolic enzyme, protein farnesyltransferase (FTase). Although the CaaX motif of progerin is intact, the 50-amino acid deletion eliminates the cleavage site for the endoprotease ZMPSTE24 (7, 9, 10); consequently, progerin cannot undergo the final endoproteolytic processing step that would normally clip off the carboxyl-terminal portion of the protein (including the farnesylated cysteine) $(1,2,7)$. Thus, farnesylated progerin accumulates in cells (7). Progerin is targeted to the nuclear rim and causes misshapen nuclei, almost certainly by interfering with the integrity of the nuclear lamina $(1,2,11-13)$.

Yang et al. (13) recently created a gene-targeted HGPS allele, $L m n a^{H G}$, which exclusively yielded progerin. Heterozygous mice $\left(L m n a^{H G /+}\right)$ exhibit a variety of phenotypes similar to those in humans with HGPS (e.g., normality at birth followed by slow

Nonstandard abbreviations used: AG, 8-anilinogeraniol; AGPP, anilinogeranyl diphosphate; FTase, farnesyltransferase; FTI, FTase inhibitor; HGPS, HutchinsonGilford progeria syndrome; MEF, mouse embryonic fibroblast.

Conflict of interest: The authors have declared that no conflict of interest exists. Citation for this article: J. Clin. Invest. 118:3291-3300 (2008). doi:10.1172/JCI35876. growth, osteoporosis, alopecia, partial lipodystrophy) (14). Like fibroblasts from children with $\operatorname{HGPS}(1,2), \mathrm{Lmna}^{\mathrm{HG} /+}$ mouse embryonic fibroblasts (MEFs) have misshapen nuclei (13). We hypothesized that protein farnesylation could be important for disease pathogenesis (15) and further hypothesized that inhibiting protein farnesylation with a FTase inhibitor (FTI) might reduce the frequency of misshapen nuclei and ameliorate the disease phenotypes in $L m n a^{H G /+}$ mice. Indeed, an FTI lowers the frequency of misshapen nuclei in $\mathrm{Lmna}^{\mathrm{HG} /{ }^{+}}$MEFs (13). Also, in 2 independent studies $(14,16)$, a highly selective FTI reduces disease phenotypes in $L m n a^{H G /+}$ mice. An FTI also reduces disease phenotypes in ZMPSTE24-deficient mice (17), where the farnesylated form of wildtype prelamin A accumulates $(9,10)$. While an FTI was unequivocally efficacious in all 3 studies $(14,16,17)$, the amelioration of disease was far from complete; all of the FTI-treated mice ultimately developed severe phenotypes and succumbed to the disease.

The improved disease phenotypes in $L m n a^{H G /+}$ mice treated with an FTI have prompted a clinical trial of an FTI treatment regimen in children with HGPS $(18,19)$. Given the merciless nature of this disease, we hope that the FTI treatment will be efficacious. However, key questions remain about the scientific underpinnings of the FTI strategy. An FTI will leave HGPS cells with a distinctly abnormal lamin protein, nonfarnesylated progerin, and it is unclear whether that protein might retain the ability to elicit disease. An optimist might contend that nonfarnesylated progerin would be entirely nontoxic, given that FTIs can markedly reduce the frequency of misshapen nuclei in HGPS fibroblasts $(13,20,21)$. On the other hand, a pessimist would point to the less-than-complete amelioration of disease phenotypes in FTI-treated $L m n a^{H G /+}$ mice $(14,16)$ and suggest that nonfarnesylated progerin might retain the capacity to cause disease.

The standard approach for assessing the functional importance of protein farnesylation is to examine the properties of a nonfarne- 


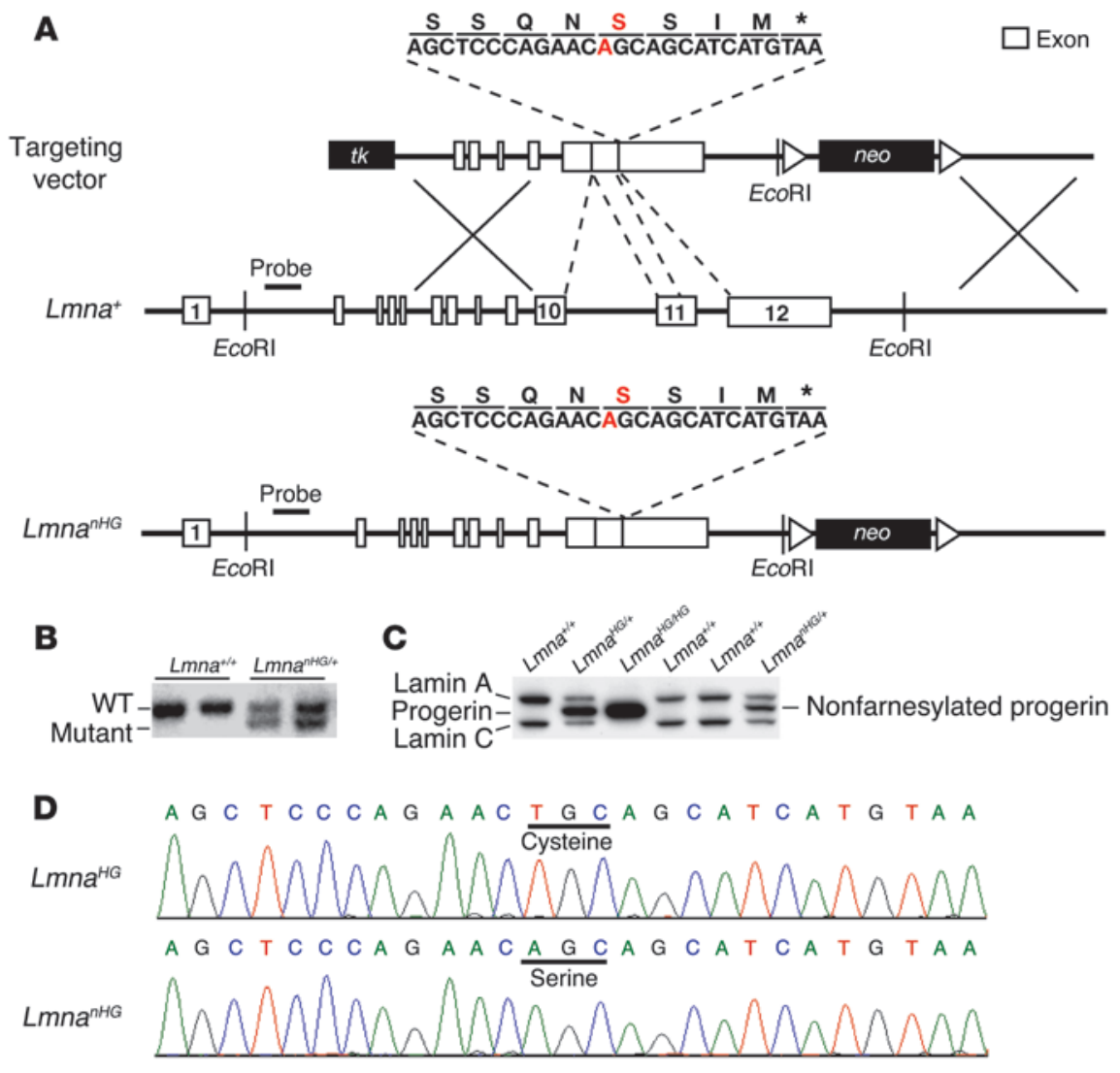

\section{Figure 1}

Production of mice that express a nonfarnesylated version of progerin. (A) The mutant allele yielding nonfarnesylated progerin, Lmnan ${ }^{n H G}$, was generated by deleting intron 10 , intron 11 , and the last $150 \mathrm{bp}$ of exon 11 and introducing a point mutation in exon 12 that changes the cysteine of the CaaX motif to a serine. (B) Southern blot identification of the targeting event in 2 different mouse ES cell clones. The genomic DNA was cleaved with EcoRI, and the blot was hybridized with a 5' flanking probe (location of probe shown in A). (C) Western blot identification of progerin in extracts of $L m n a^{n H G /+}$ MEFs with a lamin A/C-specific polyclonal antibody. Extracts of $L m n a^{+/+}$, $\mathrm{Lmna} \mathrm{HG}^{\mathrm{H} /+}$, and $\mathrm{Lmna} \mathrm{a}^{H G / H G}$ MEFs were included as controls. (D) Sequencing chromatograms of PCR products amplified from the mutant alleles of $L m n a^{H G /+}$ and $L m n a^{n H G /+}$ mice. The single nucleotide substitution in the $L m n a^{n H G}$ allele (a thymine to adenine substitution) changes the cysteine of the CaaX motif to a serine. sylated mutant protein in which the cysteine of the CaaX motif has been replaced with a serine (22-24). Serine is identical to cysteine, except that a side-chain sulfur atom is replaced with an oxygen. In the current study, we applied this approach and created a new line of HGPS mice expressing a nonfarnesylated version of progerin. Aside from the point mutation in the CaaX motif, the new genetargeted HGPS mice (Lmna $a^{n H /+}$ ) were identical to the original mouse model $\left(L m n a^{H G /+}\right)$. We went on to compare the phenotypes of genetically identical $L m n a^{n H G /+}$ and $L m n a^{H G /+}$ mice.

\section{Results}

Production of Lmna ${ }^{n H G}$ mice. We constructed a gene-targeting vector to create a mutant Lmna allele, $L m n a^{n H G}$, designed to produce nonfarnesylated progerin (Figure 1A). The Lmna ${ }^{n H G}$ allele is identical to the $L m n a^{H G /+}$ allele created earlier (13), except that the cysteine within the CaaX motif was replaced with a serine (which prevents protein farnesylation). Two targeted ES cell clones were identified by Southern blotting (Figure 1B) and used to create male chimeric mice, which were bred with C57BL/ 6 females to produce $L m n a^{n H G /+}$ mice. $L m n a^{n H G /+}$ MEFs produced progerin from the $L m n a^{n H G}$ allele and lamins A and C from the wild-type allele (Figure 1C). The electrophoretic mobility of progerin in $L m n a^{n H G /+}$ MEFs was slightly slower than in $L m n a^{H G /+}$ MEFs, which was predicted from the difference in farnesylation (Figure 1C). The point mutation in the $L m n a^{n H G}$ allele was verified by DNA sequencing (Figure 1D).

The absence of farnesylation in the progerin of $\mathrm{Lmna}^{n \mathrm{HG} /+}$ MEFs was verified by metabolically labeling with anilinogeranyl diphosphate (AGPP), a farnesyl pyrophosphate analog (25-27).
The incorporation of the AGPP into proteins can be monitored by Western blotting with an antibody against the anilinogeranyl moiety of AGPP (Figure 2A). As expected, farnesylated progerin was detected in $L m n a^{H G /+}$ but not $L m n a^{n H G /+}$ MEFs (Figure 2A). Lamins B1 and B2 are farnesylated proteins with an electrophoretic mobility identical to that of progerin (intermediate between lamins A and C). Thus, it was not surprising to observe a farnesylated protein between lamin $\mathrm{A}$ and lamin $\mathrm{C}$ in all of the MEFs (Figure 2A). However, following immunoprecipitation of the A-type lamins (i.e., lamin A, lamin C, and progerin), farnesylated progerin was observed only in $\mathrm{Lmna}^{\mathrm{HG} /{ }^{+}}$MEFs (Figure 2A).

The farnesylation of progerin in $L m n a^{H G /+}$ MEFs was abolished by treating the cells with an FTI (Figure 2A). Western blots with an antibody against HDJ-2 provided further evidence that the FTI was active (Figure $2 \mathrm{~B}$ ).

Lmna ${ }^{n H G /+}$ mice develop severe disease phenotypes. Lmna ${ }^{n H G /+}$ mice developed the same spectrum of disease phenotypes observed in $L m n a^{H G /+}$ mice, but the disease phenotypes were less severe (14). Body-weight curves were abnormal in both male and female $L m n a^{n H G /+}$ mice, compared with wild-type control mice $(P<0.0001$ for both males and females) (Figure 3, A and B). However, the growth of $L m n a^{n H G /+}$ mice was substantially better than that of $L m n a^{H G /+}$ mice $(P<0.0001$ for both males and females) (Figure 3, A and B).

Kaplan-Meier survival curves revealed that $L m n a^{n H G /+}$ mice lived longer than $L m n a^{H G /+}$ mice ( $P<0.0001$ for both males and females) (Figure $3 \mathrm{C}$ ). $\mathrm{Lmna}^{\mathrm{HG} / \mathrm{+}}$ mice develop progressive rib fractures near the costovertebral joints with increasing age (14); these same bone abnormalities were observed in $L m n a^{n H G /+}$ mice. However, the number of rib fractures at the time of death was lower in $L m n a^{n H G /+}$ mice 
A
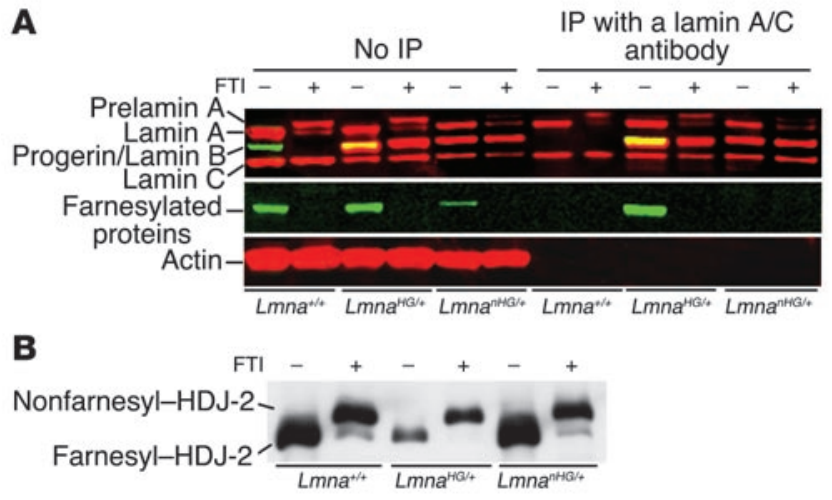

Figure 2

Assessing protein farnesylation in Lmna ${ }^{n H G /+}$ MEFs. (A) Western blot of MEF extracts performed with an Odyssey Infrared Imaging System. The top panel shows the merged images of Western blots with an antibody specific for lamin $\mathrm{A} / \mathrm{C}$ (red) and an antibody specific for the farnesol analogue AG (green); the middle panel shows the signal for the antibody against AG; the bottom panel shows a loading control (actin). In this experiment, $L m n a^{+/+}, L m n a^{H G /+}$, and $L m n a^{n H G /+}$ MEFs were incubated with $A G(30 \mu \mathrm{M})$ in the presence $(+)$ or absence $(-)$ of an FTI (ABT-100, $5 \mu \mathrm{M})$. The electrophoretic mobility of progerin, lamin B1, and lamin B2 are virtually identical, and all are farnesylated; hence, the antibody against $A G$ detected a farnesylated protein in all cell lines. When progerin and lamin $A / C$ were immunoprecipitated with an antibody against lamin A/C, the progerin in $L m n a^{H G /+}$ MEFs - but not $L m n a^{n H G /+}$ MEFs - stained with the antibody against AG. As expected, an FTI blocked the incorporation of AG into the lamin proteins. (B) Western blots of $L m n a^{+/+}, L m n a^{H G /+}$, and $L m n a^{n H G /+}$ MEFs with an HDJ-2specific monoclonal antibody. Most of the HDJ-2 in FTI-treated MEFs migrated more slowly, a characteristic of nonfarnesylated HDJ-2.

than in $L m n a^{H G /+}$ mice $(P<0.0001$ for both male and female mice) (Figure $3 \mathrm{D}$ ), even though the $L m n a^{n H G /+}$ mice were significantly older at the time of death $(36.1 \pm 0.69$ weeks vs. $21.3 \pm 0.84$ weeks in $L m n a^{H G /+}$ mice). $L m n a^{n H G /+}$ mice had more body fat than $L m n a^{H G /+}$ mice $(P<0.0001$ for both males and females) (Figure 3E).

The reduced number of rib fractures and reduced kyphosis of the spine in $L m n a^{n H G /+}$ mice were evident on $\mu C T$ scans (Figure 4, A-F). The bone density and cortical thickness in the ribs were greater in $L m n a^{n H G /+}$ mice than in $L m n a^{H G /+}$ mice (Figure 4, G and H).

Intercrossing $L m n a^{n H G /+}$ mice yielded $L m n a^{n H G / n H G}$ mice, which appeared normal for the first 2 weeks of life. By 4-8 weeks of age, however, male and female $L m n a^{n H G / n H G}$ mice began to lose weight and exhibited reduced amounts of subcutaneous and abdominal fat. The $L m n a^{n H G / n H G}$ mice $(n=11)$ had multiple rib fractures by 12 weeks of age, and all died by 17 weeks of age. In contrast, $L m n a^{H G / H G}$ mice die by 4 weeks of age (28). $L m n a^{n H G / n H G}$ mice were more severely affected than either $L m n a^{H G /+}$ or $L m n a^{n H G /+}$ mice.

Analysis of MEFs from Lmna ${ }^{n H G}$ mice. A hallmark of $L m n a^{H G /+}$ fibroblasts is misshapen nuclei. We suspected that misshapen nuclei might be less frequent in $L m n a^{n H G /+}$ MEFs than in $L m n a^{H G /+}$ MEFs. To test this possibility, we scored nuclear shape abnormalities in primary MEFs from $L m n a^{n H G /+}, L m n a^{H G /+}, L m n a^{n H G / n H G}, L m n a^{H G / H G}$, and $L m n a^{+/+}$embryos ( $n=3-8$ cell lines/genotype) (Figure 5A). These experiments involved scoring nuclear shape in 4,000 cells per genotype. In 2 independent experiments, the percentage of misshapen nuclei was lower in $L m n a^{n H G /+}$ MEFs than in $L m n a^{H G /+}$ MEFs $(P<0.0001)$ (Figure 5A). Similarly, the percentage of mis- shapen nuclei was lower in $L m n a^{n H G / n H G}$ MEFs than in $L m n a^{H G / H G}$ MEFs $(P<0.0001)$ (Figure 5A). The spectrum of nuclear shape abnormalities differed in $L m n a^{n H G /+}$ and $L m n a^{H G /+}$ MEFs; nuclei containing folds accounted for a greater percentage of the total number of misshapen nuclei in $L m n a^{n H G /+}$ and $L m n a^{n H G / n H G}$ MEFs $(P<0.0001)$ (Figure 5A).

Nuclear shape abnormalities in $L m n a^{n H G /+}, L m n a^{n H G / n H G}, L m n a^{H G /+}$, and $\mathrm{Lmna}^{+/+}$primary MEFs were examined in the presence or absence of an FTI ( $n=4$ cell lines/genotype; 4,000 cells/genotype counted) (Figure 5B). As expected, the FTI reduced the frequency of nuclear shape abnormalities in $L m n a^{H G /+}$ MEFs $(P<0.0001)$, but it had no effect on $L m n a^{n H G /+}$ or $L m n a^{n H G / n H G}$ MEFs (Figure 5B).

Normally, mature lamin A in wild-type MEFs is concentrated at the rim of the nucleus (Figure 5C). In $L m n a^{n H G / n H G} \mathrm{MEFs}$, we never observed a concentration of progerin at the nuclear rim (Figure $5 \mathrm{D})$. In many cells, the nonfarnesylated progerin was highly concentrated in nuclear folds and blebs (Figure 5, D and E).

Progerin expression levels in Lmna ${ }^{n H G /+}$ and $L m n a^{H G /+}$ mice. Disease phenotypes were less severe in $L m n a^{n H G /+}$ mice than in $L m n a^{H G /+}$ mice. To explore the potential explanation for this difference, we used Western blots to gauge progerin levels in $L m n a^{H G /+}$ and $L m n a^{n H G /+}$ mice. The levels of progerin relative to actin were lower in $L m n a^{n H G /+}$ tissues (heart, liver, kidney, skull) than in $L m n a^{H G /+}$ tissues $(P<0.0001)$ (Figure 6, A-D and F). Similarly, progerin levels relative to actin were lower in $L m n a^{n H G /+}$ MEFs than in $L m n a^{H G /+}$ MEFs $(P<0.0001)$ (Figure 6, E and F). The lower levels of progerin in $L m n a^{n H G /+}$ MEFs and tissues raised the possibility that FTI treatment might reduce the steady-state levels of progerin in $\mathrm{Lmna} \mathrm{HG}^{\mathrm{H} /+}$ MEFs and $L m n a^{H G /+}$ mice. Indeed, this was the case. Treatment of Lmna ${ }^{H G /+}$ MEFs ( $n=4$ independently isolated cell lines) with an FTI reduced progerin levels relative to actin $(P<0.0001)$ (Figure 7 , $A$ and $B)$. The FTI led to the appearance of nonfarnesylated prela$\min \mathrm{A}$, as expected, and also reduced the levels of mature lamin A. The reduced levels of mature lamin A in FTI-treated MEFs were not surprising; previously, Toth et al. (20) showed that 2 chemically distinct FTIs markedly reduced the levels of mature lamin A in wild-type MEFs.

We also analyzed progerin levels in the hearts of $L m n a^{H G /+}$ mice that had been treated with an FTI (ABT-100) for 3 or more months. The levels of progerin (relative to actin) were lower in hearts of FTI-treated $L m n a^{H G /+}$ mice than in the hearts of mice treated with vehicle alone. This difference was significant $(P<0.0001)$ (Figure 7 , $\mathrm{C}$ and D) and was observed in 2 independent experiments.

To examine the mechanism for the lower levels of progerin in $L m n a^{n H G /+}$ MEFs and tissues, we measured progerin transcript levels in $L m n a^{n H G /+}$ and $L m n a^{H G /+}$ tissues. Progerin mRNA levels, as judged by RT-PCR, were not different in the 2 mouse models (Figure 8, A and B). The fact that progerin levels were lower in Lmna ${ }^{n H G /+}$ MEFs and tissues (Figure 6) despite similar progerin transcript levels implied that the absence of farnesylation might accelerate progerin turnover. To test this possibility, we examined the disappearance of progerin in $L m n a^{n H G /+}$ and $L m n a^{H G /+}$ MEFs after labeling with $\left[{ }^{35} \mathrm{~S}\right]$ methionine. In the span of 4 days after labeling of the cells, the progerin/lamin C ratio in $L m n a^{n H G /+}$ MEFs fell by $55.6 \%$, while the progerin/lamin C ratio in $L m n a^{H G /+}$ MEFs fell by $29.2 \%$. We also examined by Western blot the progerin/actin ratios in $L m n a^{n H G /+}$ and $L m n a^{H G /+}$ MEFs before and after inhibition of Lmna expression with a mouse prelamin A-specific antisense oligonucleotide (29). Two days after the second oligonucleotide transfection, the progerin/actin ratio fell by $39.4 \%$ in 

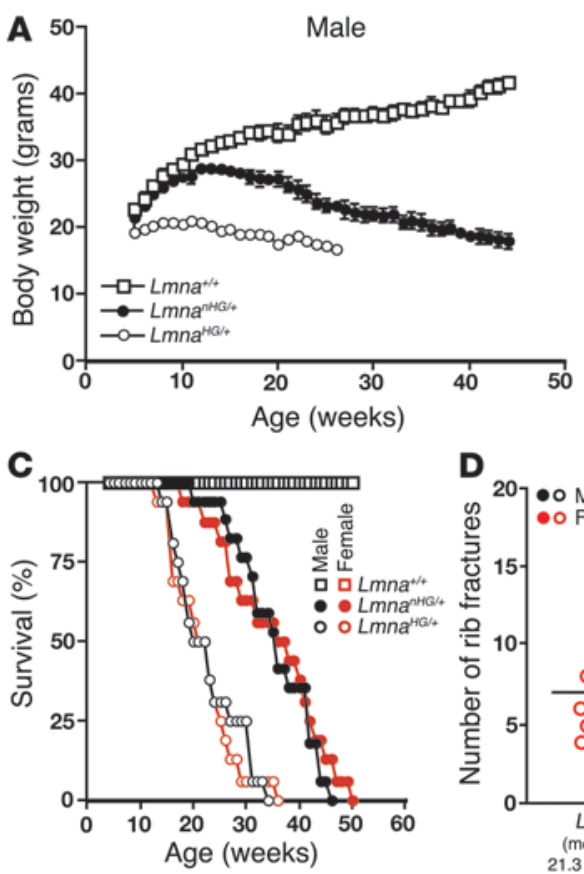
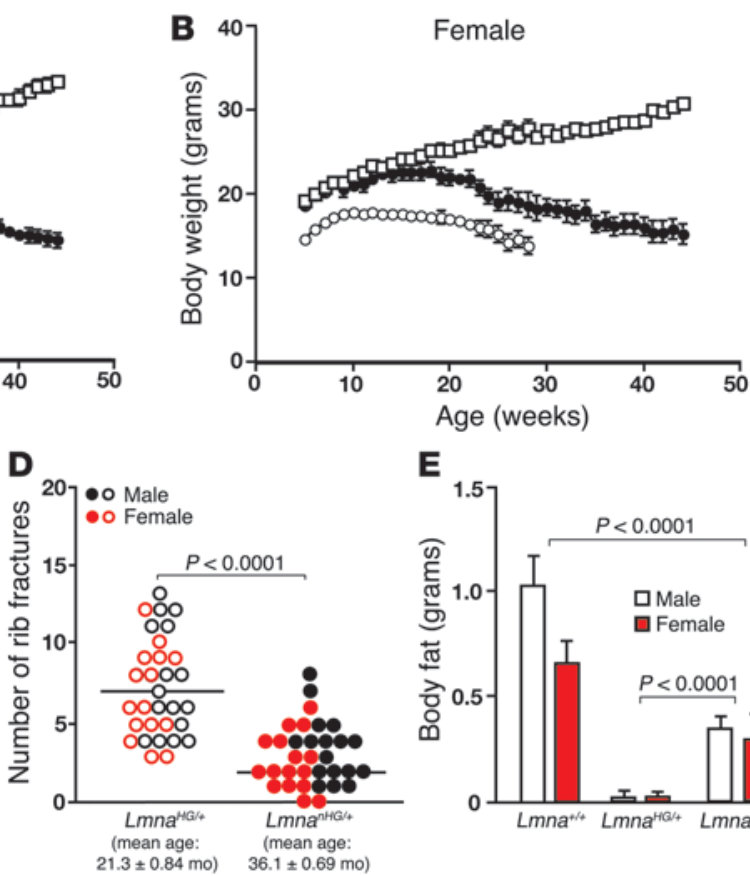

E

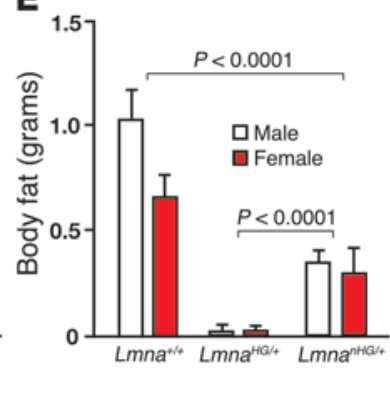

\section{Figure 3}

Phenotypes of $L m n a^{n H G /+}$ mice. (A) Body weight curves of male $L m n a^{n H G /+}(n=17), L m n a^{+/+}(n=15)$, and $L m n a^{H G /+}$ mice $(n=16)$. (B) Body weight curves of female $L m n a^{n H G /+}(n=16), L m n a^{+/+}(n=12)$, and $L m n a^{H G /+}$ mice $(n=15)$. Body weights were lower in male and female $L m n a^{n H G /+}$ mice than in $L m n a^{+/+}$mice $(P<0.0001)$. However, body weights were higher in male and female $L m n a^{n H G /+}$ mice than in $L m n a^{H G /+}$ mice $(P<0.0001)$. (C) Kaplan-Meier survival plots for $L m n a^{n H G /+}$ mice ( $n=17$ males, 16 females), $L m n a^{H G /+}$ mice ( $n=16$ males, 15 females), and $L m n a^{+/+}$mice ( $n=15$ males, 12 females). Male and female $L m n a^{n H G /+}$ mice survived longer than $L m n a^{H G /+}$ mice $(P<0.0001)$. (D) Number of rib fractures in $\mathrm{Lmna}^{n \mathrm{HG} /+}$ mice ( $n=17$ males, 16 females) and $\mathrm{Lmna}{ }^{\mathrm{HG} /+}$ mice ( $n=16$ males, 15 females). Rib fractures normally increase with age (14). The number of fractures was lower in $L m n a^{n H G /+}$ mice than in $L m n a^{H G /+}$ mice $(P<0.0001)$, even though $L m n a^{n H G /+}$ mice were older than the $L m n a^{H G /+}$ mice $\left(36.1 \pm 0.69\right.$ weeks vs. $21.3 \pm 0.84$ weeks). (E) Body fat in $L m n a^{+/+}, L m n a^{H G /+}$, and $L m n a^{n H G /+}$ mice at the conclusion of the study. Body fat in $L m n a^{n H G /+}$ mice was lower than in $L m n a^{+/+}$mice $(P<0.0001)$ but higher than in $L m n a^{H G /+}$ mice $(P<0.0001)$. Error bars indicate SEM.

Lmna ${ }^{H G /+}$ MEFs and by $58.9 \%$ in $L m n a^{n H G /+}$ MEFs. These observations are consistent with the lower steady state levels of progerin in $L m n a^{n H G /+}$ MEFs and tissues (Figure 6).

\section{Discussion}

In 2 independent studies $(14,16)$, an FTI ameliorated disease phenotypes in $L m n a^{H G /+}$ mice. However, the amelioration of disease was far from complete, leading us to consider the possibility that the nonfarnesylated form of progerin might itself be toxic and capable of eliciting disease. In the current study, we created a new line of HGPS-knockin mice expressing a nonfarnesylated version of progerin $\left(\mathrm{Lmna}^{n H G /+}\right)$. Aside from a cysteine-to-serine substitution in the CaaX motif, $L m n a^{n H G /+}$ mice were genetically identical to $L m n a^{H G /+}$ mice (13). The absence of progerin farnesylation in $L m n a^{n H G /+}$ cells was verified with metabolic labeling experiments. Interestingly, the $L m n a^{n H G /+}$ mice developed the same spectrum of

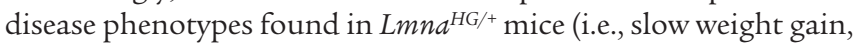
rib fractures, loss of body fat, reduced survival). Thus, the principal message of this study is that a nonfarnesylated version of progerin is still toxic. However, the extent of disease phenotypes in $L m n a^{n H G /+}$ mice was milder than in $L m n a^{H G /+}$ mice. Also, the disease phenotypes in $L m n a^{n H G / n H G}$ mice were milder than those in $L m n a^{H G / H G}$ mice (14). Thus, the second message of this study is that disease phenotypes are milder in the knockin mice expressing a nonfarnesylated version of progerin. The finding of less severe phenotypes in $L m n a^{n H G /+}$ mice is consistent with the results of earlier FTI treatment studies $(14,16)$.

Both $L m n a^{n H G /+}$ and $L m n a^{H G /+}$ mice produced progerin, and they were genetically identical - except at a single codon. However, the severity of the phenotypes was reduced in $L m n a^{n H G /+}$ mice. We reasoned that one explanation might be lower steady-state levels of progerin in $L m n a^{n H G /+}$ mice. Indeed, the levels of progerin, both in MEFs and tissues, were significantly lower in $L m n a^{n H G /+}$ mice than in $L m n a^{H G /+}$ mice despite similar levels of progerin transcripts, and follow-up studies in MEFs pointed to a more rapid disappearance of nonfarnesylated progerin. Reduced levels of progerin in the absence of farnesylation were not entirely surprising, as there are precedents for the absence of prenylation causing reduced steady-state levels of other CaaX proteins $(30,31)$. It is quite plausible that the different steady-state levels of progerin in $L m n a^{n H G /+}$ and $L m n a^{H G /+}$ mice could explain the phenotypic differences in the 2 models. Higher levels of progerin expression are associated with more nuclear shape abnormalities (11), and rare HGPS mutations yielding higher-than-usual progerin levels are associated with particularly severe forms of progeria (32). Also, we found that $L m n a^{H G / L C O}$ mice (compound heterozygotes with $1 \mathrm{Lmna}^{\mathrm{HG}}$ allele and 1 "lamin C-only" allele) have lower steady-state levels of progerin than $\mathrm{Lmna}^{\mathrm{HG} / \mathrm{+}}$ mice, despite identical levels of progerin transcripts. The reduced progerin levels in $L m n a^{H G / L C O}$ mice were accompanied by reduced disease phenotypes and a reduced frequency of misshapen nuclei (28). 

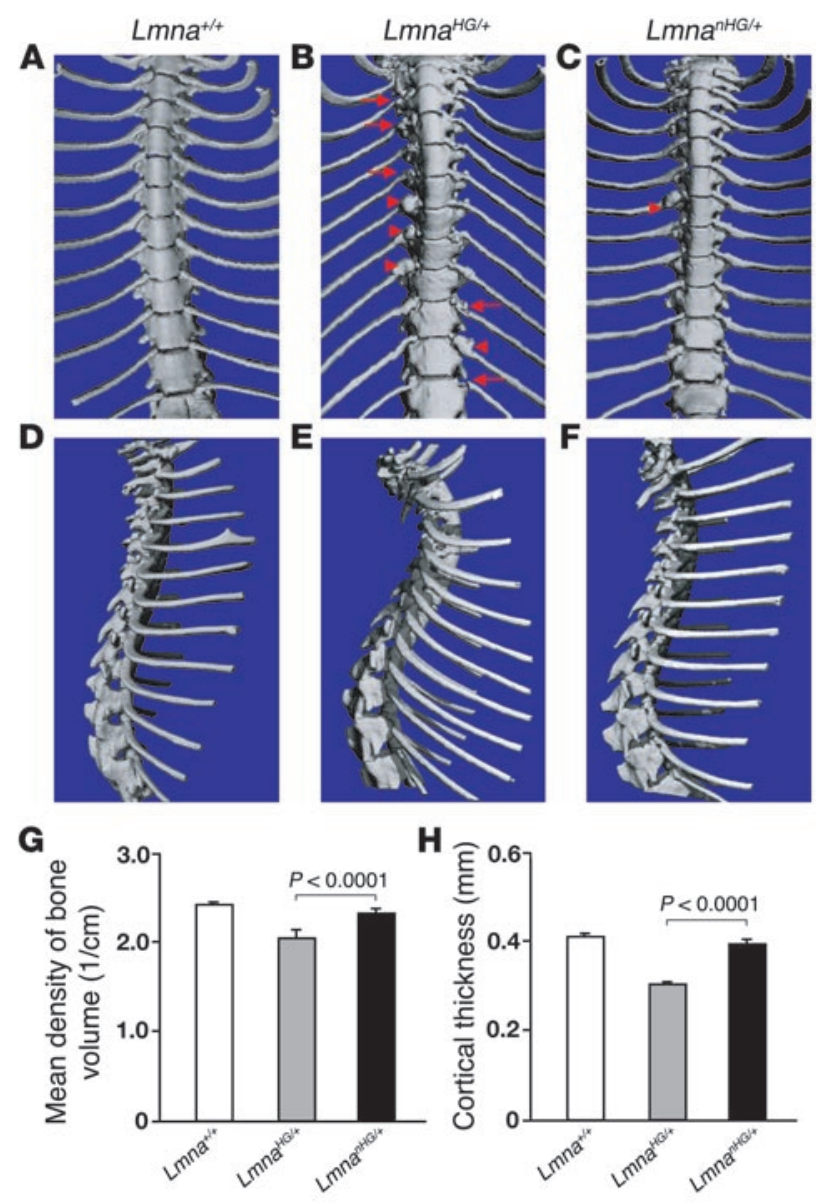

Given the very positive effects of FTIs on nuclear shape in HGPS cells $(13,20,21,33,34)$, many will probably be quite surprised by the fact that nonfarnesylated progerin is toxic. However, viewed from the perspective of the human genetics of progeroid syndromes, one could argue that the findings are not particularly shocking. Very rare missense mutations in lamin A and lamin C - abnormalities that do not affect the carboxyl-terminal processing of prelamin A - can cause progeroid disorders in humans. For example, E145K and S143F mutations in lamins A/C clearly elicit progeria-like disease phenotypes $(1,35)$, and compound heterozygosity for T528M and M540T substitutions in lamins A/C causes a progeroid syndrome that is similar to HGPS (36). Why these subtle structural variations cause progeroid syndromes rather than other "lamin A/C diseases" such as lipodystrophy or muscular dystrophy is not known, but at face value these genetic observations suggest that protein farnesylation is not "the entire story" in the pathogenesis of progeroid syndromes. Our current studies with nonfarnesylated progerin reinforce that view.

In our study, we eliminated protein farnesylation with the standard approach, which is to replace the cysteine of the CaaX motif with a serine (22-24). The potential impact of a single amino acid substitution in lamin A or lamin C must be considered carefully, given that single amino acid substitutions in these proteins can elicit disease in humans (4). Indeed, one could argue that our conclusions require a caveat. The nonfarnesylated progerin in $\mathrm{Lmna}^{n \mathrm{HG} /+}$ mice contains a missense mutation. One might conceivably argue - and it is clearly a formal possibility - that nonfarne-

\section{Figure 4}

Reduced bone abnormalities in $L m n a^{n H G /+}$ mice at 6 months of age, as judged by surface renderings of $\mu \mathrm{CT}$ analyses. (A-F) $\mu \mathrm{CT}$ scans of the thoracic spine illustrating reduced number of rib fractures in $\mathrm{Lmna} \mathrm{a}^{n \mathrm{HG} /+}$ mice. Red arrowheads indicate rib fractures and surrounding callus. Red arrows indicate thinning ribs along with a small amount of callus. (A and D) $L m n a^{+/+}$mouse; (B and E) $L m n a^{H G /+}$ mouse; (C and F) $L m n a^{n H G /+}$ mouse. (D-F) Lateral view of the thoracic spine illustrating reduced kyphosis of the spine in $L m n a^{n H G /+}$ mouse. Bone density (G) and cortical thickness $(\mathbf{H})$ were improved in the ribs of $L m n a^{n H G /+}$ mice compared with $L m n a^{H G /+}$ mice ( $n=4$ mice/genotype; $\left.P<0.0001\right)$. Error bars indicate SEM.

sylated progerin with a cysteine in the CaaX motif (as would occur during FTI treatment of $\mathrm{Lmna}^{\mathrm{HG} / \mathrm{+}}$ mice) is $100 \%$ innocuous but that a nonfarnesylated progerin with the cysteine-to-serine substitution (as in $L m n a^{n H G /+}$ mice) is toxic and elicits disease phenotypes similar to those associated with farnesylated progerin in untreated $L m n a^{H G /+}$ mice. According to this reasoning, it is the point mutation in the Lmna ${ }^{n H G}$ allele and not the 50-amino acid deletion that causes disease. There is no good experimental way to exclude this possibility, but this seems quite unlikely. First, the 50-amino acid deletion is a far more substantial structural alteration than a cysteine-to-serine substitution. Second, there is no precedent for a heterozygous Lmna missense mutation eliciting disease phenotypes in mice. Heterozygosity for L530P, H222P, and N195K mutations causes severe disease in humans (37-39) but no disease phenotypes in mice (40-42).

Based on the efficacy of FTI treatment in $\mathrm{Lmna}^{\mathrm{HG} /{ }^{+}}$and $\mathrm{Zmpste24^{-/- }}$ mice $(14,16,17)$, a clinical trial of FTI therapy is under way in children with HGPS $(18,19)$. What are the implications of our current studies for the treatment of HGPS? On the positive side, our current studies are consistent with our FTI treatment results $(14,16,17)$ and suggest that reducing progerin farnesylation might be useful. On the other hand, our current studies suggest that the impact of an FTI could be limited. Despite a total absence of progerin farnesylation, $L m n a^{n H G /+}$ mice still developed severe disease phenotypes. Thus, while FTIs may well ameliorate some phenotypes in children with HGPS, we doubt that FTIs will be a panacea, and we are skeptical that other strategies to interfere with protein prenylation, such as bisphosphonates (20), will be any better. We believe that it will be important to continue to develop other therapeutic strategies, such as approaches to reduce the alternative splicing event that lies at the root of the disease (12), or to eliminate prelamin A transcripts with antisense approaches $(29,43)$.

\section{Methods}

Knockin mice expressing nonfarnesylated progerin. The targeting vector for the $L m n a^{n H G}$ allele is identical to that of the $L m n a^{H G}$ allele produced previously (13), except for a point mutation in exon 12 (in the $5^{\prime}$ arm of the vector) that changes the cysteine of the CaaX motif to a serine. The mutation was introduced into a plasmid containing the $5^{\prime}$ arm of the vector by site-directed mutagenesis with the QuikChange kit (Stratagene) with primer 5'-CAGAGCTCCCAGAACAGCAGCATCATGTAATCT-3' (the single-base change is underlined) and a complementary reverse primer. The integrity of the completed gene-targeting vector was verified by restriction endonuclease digestion (the point mutation eliminates a Pst site) and DNA sequencing (13).

Targeted 129/OlaHsd ES cell clones carrying the $L m n a^{n H G}$ allele were identified by Southern blotting with EcoRI-cleaved genomic DNA and a 


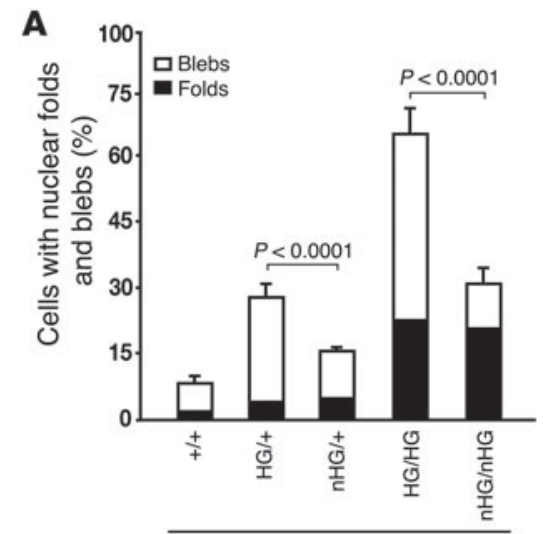

C

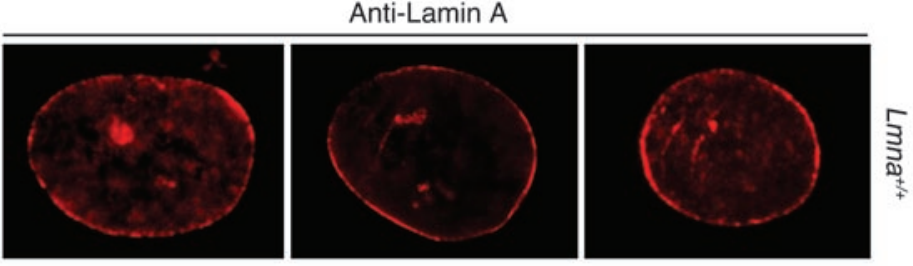

D

Anti-Lamin A
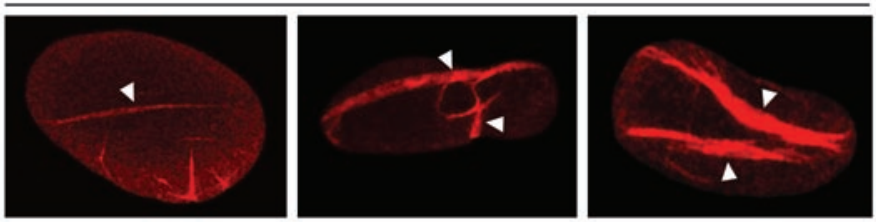

Genotype
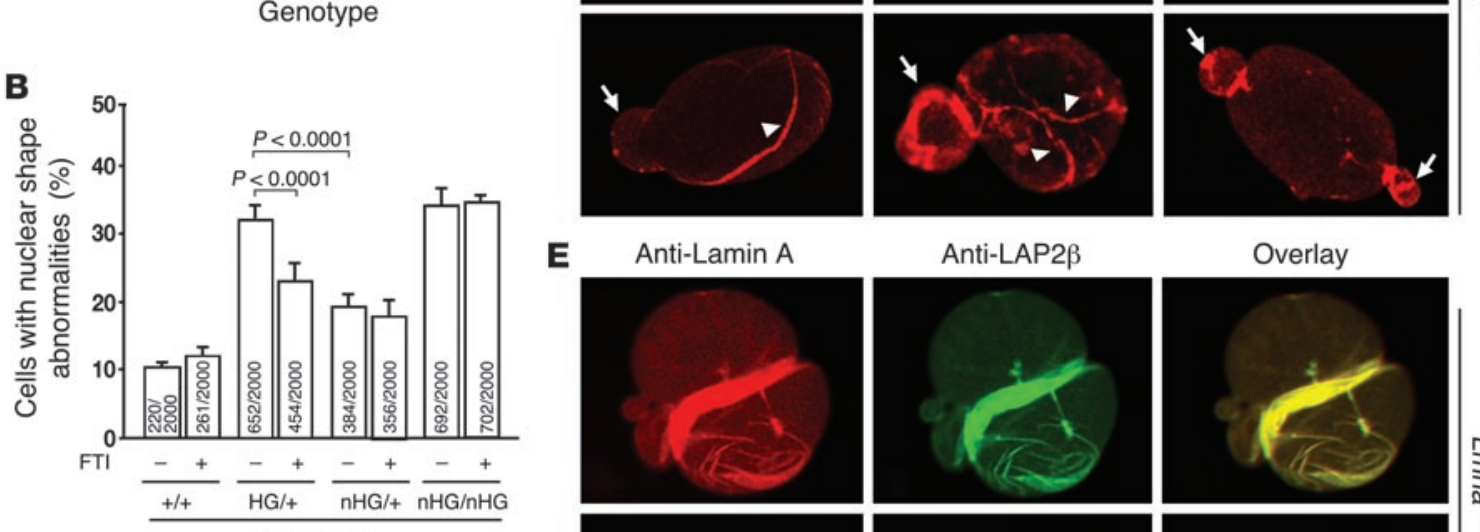

E
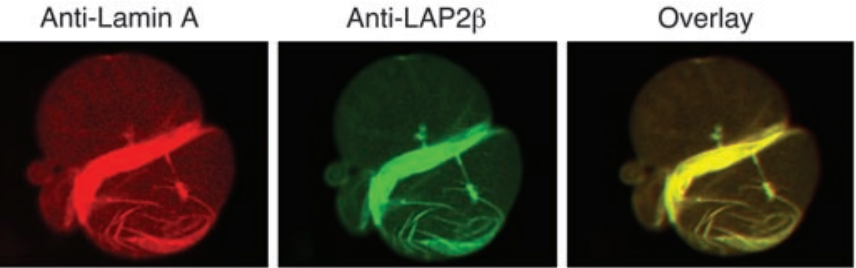

Genotype
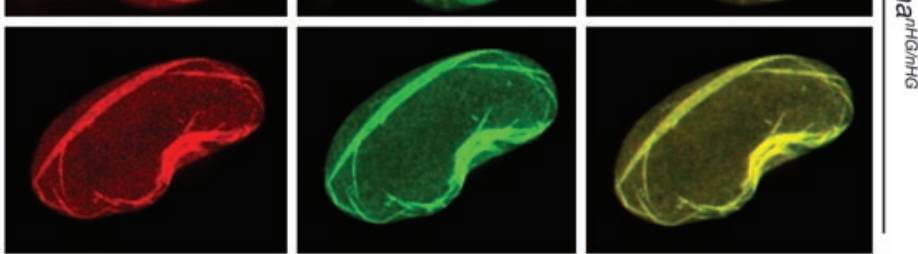

\section{Figure 5}

Analysis of nuclear shape in primary MEFs from $L m n a^{+/+}, L m n a^{n H G /+}, L m n a^{n H G / n H G}, L m n a^{H G /+}$, and $L m n a^{H G / H G}$ embryos by immunofluorescence microscopy. (A) The frequency of misshapen nuclei (folds, black bars; blebs, white bars) was greater in $L m n a^{n H G /+}$ and $L m n a^{n H G / n H G}$ MEFs than in $L m n a^{+/+}$MEFs, but the frequency of misshapen nuclei was lower in $L m n a^{n H G /+}$ and $L m n a^{n H G / n H G}$ MEFs than in $L m n a^{H G /+}$ and $L m n a^{H G / H G} \mathrm{MEFs}$, respectively ( $n=3-8$ cell lines/genotype, $>1000$ cells counted for 3 fibroblast cell lines of each genotype; $P<0.0001, \chi^{2}$ test). Error bars indicate SEM for results with independently isolated cells of the same genotype. The fraction of abnormal nuclei that had nuclear folds was higher in Lmna ${ }^{n H G /+}$ MEFs than in $L m n a^{H G /+}$ MEFs and higher in $L m n a^{n H G / n H G}$ MEFs than in $L m n a^{H G / H G}$ MEFs $(P<0.0001)$. (B) Frequency of misshapen nuclei in $\mathrm{Lmna}^{+/+}, \mathrm{Lmna} \mathrm{HG}^{\mathrm{H} /+}$, and $L m n a^{n H G /+}$ MEFs in the presence and absence of an FTI (10 $\left.\mu \mathrm{M}, \mathrm{ABT}-100\right)$. The FTI reduced the number of misshapen nuclei in $L m n a^{H G /+}$ MEFs, and an FTI had no effect on $L m n a^{+/+}$MEFs $\left(n=4\right.$ cell lines/genotype; $P<0.0001, \chi^{2}$ test). In $L m n a^{n H G /+}$ and $L m n a^{n H G / n H G}$ MEFs, the FTI treatment had no significant effect on nuclear shape ( $n=4$ cell lines/genotype; 4,000 cells/genotype counted). Ratios in each bar represent the number of cells with misshapen nuclei divided by the total number of cells evaluated (from all cell lines of each genotype). Error bars indicate SEM. Similar results, with identical levels of statistical significance, were obtained by 3 independent observers - all blinded to genotype. (C) Immunostaining of wild-type MEFs with a lamin A-specific antibody (red). (D) Immunostaining of $L m n a^{n H G / n H G ~ M E F s ~ w i t h ~ a ~ l a m i n ~ A-s p e c i f i c ~}$ antibody (red). Blebs are indicated by white arrows, and folds are indicated by white arrowheads. (E) The localization of progerin (red) and LAP2 $\beta$ (green) in $L m n a^{n H G / n H G}$ MEFs; both proteins were concentrated in nuclear folds. Images were recorded with a $\times 63$ oil immersion objective (C-E).

348-bp 5' flanking probe (13). The probe detects a 10.4-kb band in the wild-type Lmna allele and a $9.3-\mathrm{kb}$ band in the $L m n a^{n H G}$ allele. To produce chimeric mice, targeted ES cells were microinjected into C57BL/6 blastocysts. Chimeras were bred with $\mathrm{C} 57 \mathrm{BL} / 6$ females to generate $L m n a^{n H G /+}$ mice. Chimeras generated from $L m n a^{H G /+}$ ES cells were used to generate $L m n a^{H G /+}$ mice. Because $L m n a^{n H G /+}$ and $L m n a^{H G /+}$ mice were bred from chimeras, they were genetically identical (with $1 \mathrm{C} 57 \mathrm{BL} / 6$ chromosome and 1 129/OlaHsd chromosome) except for the point mutation in exon 12 of the $L m n a^{n H G}$ allele. $L m n a^{n H G /+}$ mice were intercrossed to obtain $L m n a^{n H G / n H G}$ mice.
Genotyping of mice was performed by PCR with genomic DNA from tail biopsies and oligonucleotide primers 5'-TGAGTACAACCTGCGCTCAC-3' and 5'-CAGACAGGAGGTGGCATGT-3'. The wild-type allele yields a 582-bp product, whereas the mutant allele yields a 186-bp product (13).

In some experiments, $L m n a^{H G /+}$ mice were treated with an FTI ABT-100 (14). ABT-100 was kindly provided by David J. Frost (Abbott). The FTI was mixed in drinking water containing $0.4 \%$ hydroxy propyl methyl cellulose and $1.0 \%$ ethanol at a concentration of $0.3 \mathrm{mg} / \mathrm{ml}$, so as to deliver a dose of approximately $39 \mathrm{mg} / \mathrm{kg} / \mathrm{d}$. The vehicle control consisted of drinking water with $0.4 \%$ hydroxy propyl methyl cellulose and $1.0 \%$ ethanol (14). 
A

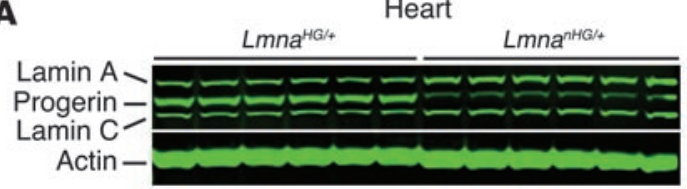

B

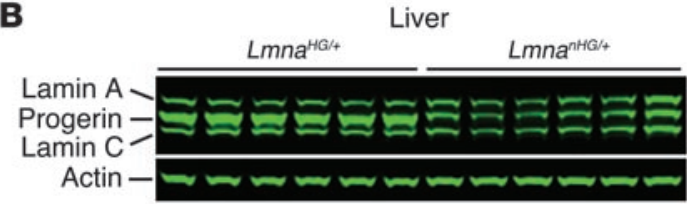

C

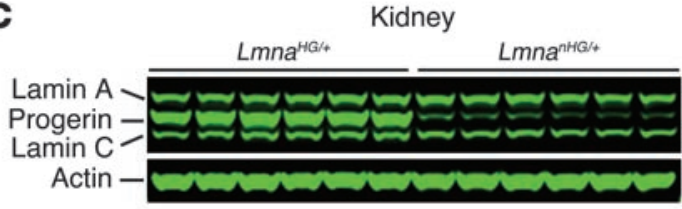

D

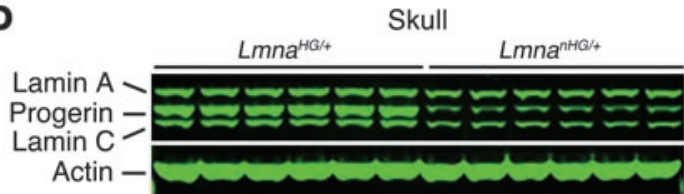

E

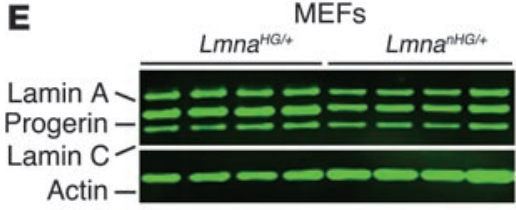

$\mathbf{F}$

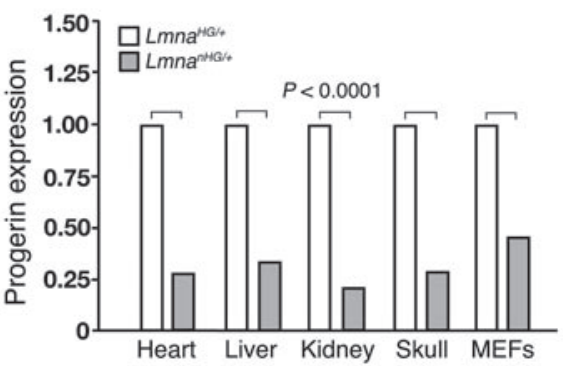

\section{Figure 6}

Western blots documenting relative levels of progerin in $L m n a^{H G /+}$ and $L m n a^{n H G /+}$ tissues and MEFs. (A-D) Western blot (with antibodies against lamin A/C and actin) showing progerin, lamin A, lamin C, and actin in the heart (A), liver (B), kidney (C), and skull (D) of $L m n a^{H G /+}$ and $L m n a^{n H G /+}$ mice ( $n=3 /$ genotype; each sample loaded in duplicate). (E) Western blot analysis (with antibodies against lamin A/C and actin) showing progerin, lamin A, lamin C, and actin in $L m n a^{H G /+}$ and $L m n a^{n H G /+}$ MEFs ( $n=4$ cell lines/genotype). (F) Quantitative analysis of progerin expression in the tissues ( $n=3$ mice/genotype) and MEFs ( $n=4$ cell lines/genotype) of $L m n a^{H G /+}$ and $L m n a^{n H G /+}$ mice. Each sample was analyzed on 4 separate Western blots; the variation in the progerin/actin ratio averaged $5.6 \%$. Progerin/actin ratios in $L m n a^{n H G /+}$ samples were expressed relative to those in $L m n a^{H G /+}$ samples (which were set at a value of 1 ). In both mouse tissues and the MEFs, the progerin/actin ratio was lower in $L m n a^{n H G /+}$ mice than in $L m n a^{H G /+}$ mice $(P<0.0001)$.

All mice were fed a chow diet and housed in a virus-free barrier facility with a 12-hour light/12-hour dark cycle. Body weights were assessed weekly. All animal studies were approved by UCLA's Animal Research Committee.

Cell culture. Primary MEFs were prepared from E13.5 embryos (10). Earlypassage MEFs were plated in 6-well plates and grown to $75 \%$ confluency. In some experiments, protein farnesylation was blocked by incubating the cells with an FTI (10 $\mu \mathrm{M}$ ABT-100) for 48 hours.

Metabolic labeling to detect protein farnesylation. MEFs were incubated for 36 hours with an analogue of farnesol, 8-anilinogeraniol (AG) $(30 \mu \mathrm{M}$ in DMSO) (25), in the presence and absence of an FTI (ABT-100, $5 \mu \mathrm{M})$. After entering cells, AG is incorporated into AGPP, which is used by protein FTase as a substrate for the modification of $\mathrm{CadX}$ proteins such as progerin. The AG moiety of AGPP is transferred onto cellular proteins by FTase, competitive with endogenous pools of farnesyl diphosphate (25). AG incorporation into endogenous cellular proteins was detected by Western blotting with a mouse monoclonal antibody specific for AG, diluted 1:5000 (25).

Immunoprecipitation. MEFs were washed with PBS and harvested by scraping, then pelleted by centrifugation. The pellet was resuspended in $0.3 \mathrm{ml}$ ice-cold lysis buffer (50 mM Tris, $0.15 \mathrm{M} \mathrm{NaCl}, 1 \mathrm{mM}$ EDTA, $1 \%$ NP-40, $2.5 \mathrm{mg} / \mathrm{ml}$ deoxycholate, $1 \mathrm{mM}$ PMSF, and $0.1 \%$ SDS in PBS) containing Complete Mini Protease Inhibitors (Roche) at $1 \times$ concentration, and incubated on ice for 10 minutes. After removing insoluble material by centrifugation, cell extracts were precleared by incubation with $25 \mu$ l of protein Gagarose (50\% suspension in lysis buffer) (Roche) at $4^{\circ} \mathrm{C}$ for 30 minutes. Goat anti-lamin A/C antibody (15 $\mu \mathrm{l}$ ) (Santa Cruz Biotechnology Inc.) was added to the precleared supernatant fluids and incubated at $4^{\circ} \mathrm{C}$ overnight with constant rotation. Immune complexes were isolated by the addition of protein $\mathrm{G}$-agarose and incubation at $4^{\circ} \mathrm{C}$ for 2 hours, followed by centrifugation. The agarose gel was washed 5 times with ice-cold lysis buffer, and the bound proteins were released by boiling for 3 minutes in $2 \times$ sample loading buffer (Invitrogen). The agarose gel was removed by centrifugation, and the supernatant fluids were analyzed by SDS-polyacrylamide gel electrophoresis and Western blotting.

Metabolic labeling of lamins with ${ }^{35} S$ ] methionine. Lmna ${ }^{n H G /+}$ and $L m n a^{H G /+}$ MEFs were grown in methionine-deficient medium (Invitrogen) and $10 \%$ fetal bovine serum (dialyzed against PBS) at $37^{\circ} \mathrm{C}$ overnight and then labeled with $\left[{ }^{35} \mathrm{~S}\right]$ methionine $(200 \mu \mathrm{Ci} / \mathrm{ml}$; MP Biomedicals) for 24 hours. After labeling, cells were grown in standard DMEM/10\% fetal calf serum. MEFs were harvested after the labeling period and daily for 4 days. Lamin $\mathrm{A} / \mathrm{C}$, progerin, and actin were immunoprecipitated and size fractionated by SDS-PAGE. The intensities of the progerin, lamin C, and actin bands were quantified with a Typhoon 9410 Phosphorimager (GE Healthcare), and the progerin/lamin C ratio was calculated.

Protein extraction and Western blots. Urea-soluble extracts were prepared from early-passage MEFs and tissues as described previously (44). Freshly harvested mouse tissues $(\sim 150 \mathrm{mg})$ were snap-frozen in liquid nitrogen and then ground into a powder with a mortar and pestle; the powder was resuspended in $0.5 \mathrm{ml}$ of urea-solubilization buffer (44) and ground in a glass tissue grinder for 2 minutes. Finally, the samples were sonicated and centrifuged at $14,000 \mathrm{~g}$ for 10 minutes. The extracts were size fractionated on $4 \%-12 \%$ gradient polyacrylamide Bis-Tris gels (Invitrogen) and the separated proteins transferred to nitrocellulose membranes for West- 
A

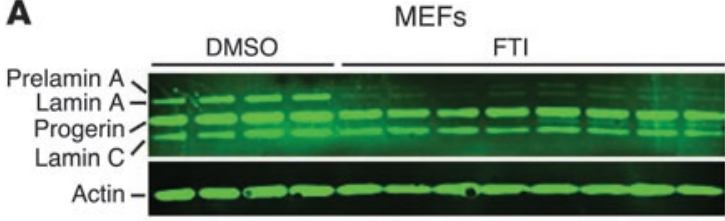

C

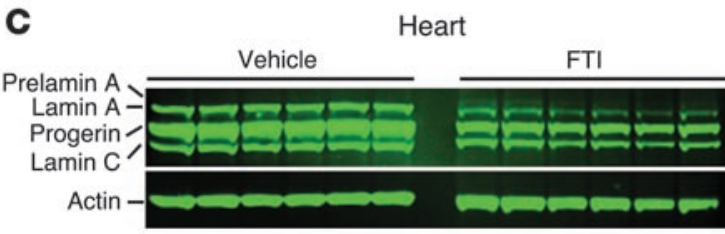

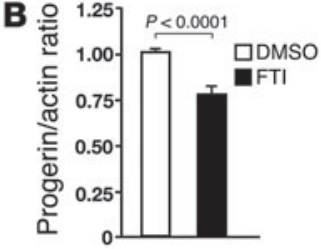

D.

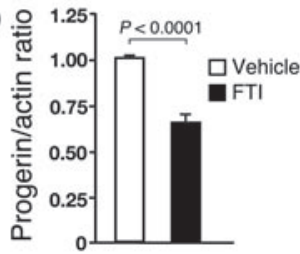

Figure 7

Western blots assessing the levels of progerin relative to actin in $L m n a^{H G /+} \mathrm{MEFs}$ and in the hearts of $L m n a^{H G /+}$ mice in the presence and absence of an FTI. (A) Western blot (with antibodies against lamin $\mathrm{A} / \mathrm{C}$ and actin) showing progerin, prelamin $\mathrm{A}$, lamin $\mathrm{A}$, lamin $\mathrm{C}$, and actin in $L m n \mathrm{~m}^{H G /+}$ MEFs ( $n=4$; the extracts of FTI-treated cells were loaded in duplicate). Cells were treated with an FTI (10 $\mu$ M, ABT-100) or vehicle (DMSO) alone for 2 weeks. (B) Quantitative analysis of progerin expression in $L m n a^{H G /+} \mathrm{MEFs}(n=4)$ relative to actin in the presence and absence of an FTI. The progerin/actin ratios in FTI-treated cell were expressed relative to those in DMSO-treated cells (where the ratio was set at 1.0). Each cell extract was analyzed in 3 independent experiments; the variation in the progerin/actin ratio averaged $10.6 \%$. The progerin/actin ratio was lower in the FTI-treated cells $(P<0.0001)$. (C) Western blot analysis showing progerin, prelamin A, lamin A, lamin $\mathrm{C}$, and actin in the hearts of $L m n a^{H G /+}$ mice treated with the FTI or vehicle alone ( $n=3 \mathrm{mice} /$ group; each sample loaded in duplicate). Mice were given the FTI (or vehicle alone) for 4 months, beginning at 4 weeks of age. (D) Quantitative analysis of progerin expression in the hearts of FTI-treated $L m n a^{H G /+}$ mice and $L m n a^{H G /+}$ mice treated with vehicle alone ( $n=3$; each sample loaded in duplicate). Progerin/actin ratios in the hearts of FTI-treated mice were expressed relative to those in vehicle-treated mice (where the ratio was set at 1.0). Each sample was analyzed in 3 independent experiments; the variation in the progerin/actin ratios averaged $11.3 \%$. The progerin/actin ratio was lower in the FTI-treated mice $(P<0.0001)$. Error bars indicate SEM.

ern blotting. The membranes were incubated in Odyssey blocking solution for 1 hour at room temperature before the addition of primary antibodies. The antibody dilutions were 1:400 for anti-lamin A/C rabbit IgG (sc-20681; Santa Cruz Biotechnology Inc.), 1:500 anti-HDJ-2 mouse IgG (NeoMarkers), 1:5000 for anti-AG mouse IgG, and 1:1000 for anti-actin goat IgG (sc-1616, Santa Cruz Biotechnology Inc.). The membranes were washed 3 times for 10 minutes each time in PBS containing $0.1 \%$ Tween 20 and then incubated with 1:5000 IRDye 700 anti-mouse IgG antibody and 1:5000 IRDye 800 anti-goat IgG antibody (Rockland Immunochemicals) in Odyssey Blocking buffer. The IR-coupled antibodies were detected on an Odyssey infrared imaging scanner and quantified according to the manufacturer's instructions.

Western blots were performed on $L m n a^{n H G /+}$ and $L m n a^{H G /+}$ MEFs after partial inhibition of Lmna expression with a mouse prelamin A-specific antisense oligonucleotide (29). Primary MEFs were plated on 6-well tissue culture plates and allowed to adhere overnight. The cells were washed with reduced serum Opti-MEM I medium (Invitrogen) and transfected with $30 \mathrm{nM}$ of the mouse prelamin A-specific antisense oligonucleotide (ISIS 359445) or a control oligonucleotide (29) using $10 \mu \mathrm{l} / \mathrm{ml}$ Lipofectin (Invitrogen) at $37^{\circ} \mathrm{C}$ for 5 hours. The medium was then replaced with fresh culture medium (DMEM/10\% FBS). The transfection was repeated the next day, and the cells were cultured for an additional 48 hours and then processed for Western blotting. The amount of progerin relative to lamin C was quantified with a LI-COR Odyssey scanner.

Immunofluorescence microscopy. Early-passage MEFs (matched for passage number) were grown on coverslips, fixed in 3\% paraformaldehyde, permeabilized with $0.2 \%$ Triton X-100, and blocked with PBS containing $10 \%$ fetal bovine serum and $0.2 \%$ bovine serum albumin (15). Cells were incubated for 1 hour with antibodies against lamin A (1:200, sc-20680; Santa Cruz Biotechnology Inc.) and LAP2 $\beta$ (1: 200, BD Biosciences - BD Transduction Laboratories). After washing, cells were incubated with 1:800 Cy3-conjugated anti-rabbit antibody (Jackson ImmunoResearch Laborato-
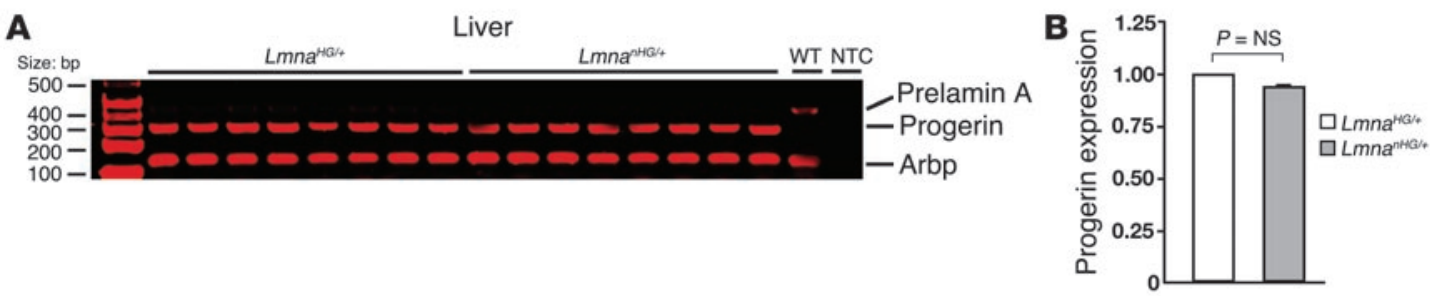

Figure 8

Progerin transcript levels in livers of $L m n a^{H G /+}$ and $L m n a^{n H G /+}$ mice $(n=4 /$ genotype). (A) Syto-60-stained agarose gel of progerin RT-PCR products (at cycle 20) from RNA of livers from $L m n a^{H G /+}$ and $L m n a^{n H G /+}$ mice $(n=4 /$ genotype, each sample run in duplicate). Image intensity was quantified with the Odyssey Infrared Imaging System. Trace amounts of wild-type prelamin A (from the wild-type allele) were observed in all samples. The RT-PCR reaction spanned Lmna exons 10-12. Arbp was used as an internal control. (B) Quantitative analysis of progerin mRNA levels, corrected for Arbp expression. Progerin mRNA levels in $L m n a^{n H G /+}$ samples were expressed relative to those in $L m n a^{H G /+}$ samples (which were set at a value of 1). Error bars indicate SEM. 
ries) and 1:600 Alexa Fluor 488-conjugated anti-mouse antibody (A21202; Molecular Probes) and DAPI to visualize DNA. Images were obtained on an Axiovert 200 MOT microscope (Zeiss) with a $\times 63 / 1.25$ oil immersion objective and processed with AxioVision 4.2 software (Zeiss). Nuclear shape $(4,000$ cells per genotype $)$ was assessed in blinded fashion as described previously $(13,20,28,29)$.

Analysis of rib fractures. The interior of the thorax of $L m n a^{+/+}, L m n a^{n H G /+}$, and $L m n a^{H G /+}$ mice was photographed with a digital camera after the removal of the heart and lung tissue, and rib fractures were counted $(14-17,28)$.

Body fat measurements. Lmna $a^{+/+}, L m n a^{H G /+}$, and $L m n a^{n H G /+}$ mice were euthanized, and the major fat pads (reproductive, inguinal, and mesenteric) were isolated and weighed.

Extraction of RNA, cDNA synthesis, and RT-PCR. The isolation of RNA, cDNA synthesis, and RT-PCR was performed exactly as described previously (28). The band intensities of the PCR fragments generated from the wild-type $L m n a$ allele and the $L m n a^{n H G /+}$ and $L m n a^{H G /+}$ alleles were quantified with the Odyssey Infrared Imaging System and the results normalized to Arbp.

Microcomputed tomographic scanning and analyses. Lmna ${ }^{+/+}, L m n a^{n H G /+}$, $L m n a^{n H G / n H G}$, and $L m n a^{H G /+}$ mice were examined by compact cone-beam tomography ( $\mu$ CT 40 scanner; SCANCO Medical). Scans were performed in the axial plane mounted in a cylindrical sample holder at medium resolution with a current of $143 \mu \mathrm{A}$ and a voltage of $55 \mathrm{kVp}$.

Bone density of isolated rib segments was determined by $\mu \mathrm{CT}$ scanning in a cylindrical sample holder tube with a voltage of $55 \mathrm{kVp}$ and current of $143 \mu \mathrm{A}$. Three-dimensional images were reconstructed in $2048 \times 2048$ pixel matrices with a standard convolution back-projection procedure with a Shepp-Logan filter, and a cortical volume of interest was defined for each measurement. The mineralized tissue was segmented with a threshold of 120 for all samples. A manufacturer-provided hydroxyapatite phantom of known density was used to calibrate the mean density of bone volume and the cortical thickness.

Statistics. Body weight curves were compared with repeated-measures ANOVA and the log-rank test. The bone density, number of rib fractures, and fat-pad weights were compared with the 2-tailed Student's $t$ test. Means and SEM are shown. Differences in the percentages of misshapen nuclei were assessed by the $\chi^{2}$ test. The number of surviving male and female mice was recorded weekly and expressed as a percentage of the total number of mice. The significance of differences was determined by the Kaplan-Meier method.

\section{Acknowledgments}

We thank B. Davies, C. Coffinier, and E. Farber for helpful discussions. We thank D. Frost for ABT-100. This study was supported by NIH grants HL76839, CA099506, and AR050200 (to S.G. Young), HL086683 (to L.G. Fong), and GM66152 to (H.P. Spielmann) and a grant from the Kentucky Lung Cancer Research Program. S.H. Yang was supported by a postdoctoral fellowship grant from the American Heart Association, Western States Affiliate, and a fellowship from the Vascular Biology Program at UCLA (supported by the NIH).

Received for publication April 8, 2008, and accepted in revised form July 16, 2008.

Address correspondence to: Stephen G. Young or Loren G. Fong, 650 Charles E. Young Dr. South, Los Angeles, California 90095, USA. Phone: (310) 825-4934; Fax: (310) 206-0865; E-mail: sgyoung@mednet.ucla.edu (S.G. Young); lfong@mednet.ucla.edu (L.G. Fong).
1. Eriksson, M., et al. 2003. Recurrent de novo point mutations in lamin A cause Hutchinson-Gilford progeria syndrome. Nature. 423:293-298.

2. de Sandre-Giovannoli, A., et al. 2003. Lamin A truncation in Hutchinson-Gilford progeria. Science. 300:2055.

3. Merideth, M.A., et al. 2008. Phenotype and course of Hutchinson-Gilford progeria syndrome. N. Engl. J. Med. 358:592-604.

4. Worman, H.J., and Bonne, G. 2007. "Laminopathies": a wide spectrum of human diseases. Exp. Cell Res. 313:2121-2133.

5. Kudlow, B.A., Kennedy, B.K., and Monnat, R.J., Jr. 2007. Werner and Hutchinson-Gilford progeria syndromes: mechanistic basis of human progeroid diseases. Nat. Rev. Mol. Cell Biol. 8:394-404.

6. Debusk, F.L. 1972. The Hutchinson-Gilford progeria syndrome. J. Pediatr. 80:697-724.

7. Young, S.G., Fong, L.G., and Michaelis, S. 2005. Prelamin A, Zmpste24, misshapen cell nuclei, and progeria - New evidence suggesting that protein farnesylation could be important for disease pathogenesis. J. Lipid Res. 46:2531-2558.

8. Lin, F., and Worman, H.J. 1993. Structural organization of the human gene encoding nuclear lamin A and nuclear lamin C. J. Biol. Chem. 268:16321-16326.

9. Pendás, A.M., et al. 2002. Defective prelamin A processing and muscular and adipocyte alterations in Zmpste24 metalloproteinase-deficient mice. Nat. Genet. 31:94-99.

10. Bergo, M.O., et al. 2002. Zmpste24 deficiency in mice causes spontaneous bone fractures, muscle weakness, and a prelamin A processing defect. Proc. Natl. Acad. Sci. U. S. A. 99:13049-13054.

11. Goldman, R.D., et al. 2004. Accumulation of mutant lamin A causes progressive changes in nuclear architecture in Hutchinson-Gilford progeria syndrome. Proc. Natl. Acad. Sci. U. S. A. 101:8963-8968.

12. Scaffidi, P., and Misteli, T. 2005. Reversal of the cellular phenotype in the premature aging disease Hutchinson-Gilford progeria syndrome. Nat. Med. 11:440-445.

13. Yang, S.H., et al. 2005. Blocking protein farnesyltransferase improves nuclear blebbing in mouse fibroblasts with a targeted Hutchinson-Gilford progeria syndrome mutation. Proc. Natl. Acad. Sci. U. S. A. 102:10291-10296.

14. Yang, S.H., et al. 2006. Treatment with a protein farnesyltransferase inhibitor improves disease phenotypes in mice with a targeted HutchinsonGilford progeria syndrome mutation. J. Clin. Invest. 116:2115-2121.

15. Fong, L.G., et al. 2004. Heterozygosity for Lmna deficiency eliminates the progeria-like phenotypes in Zmpste24-deficient mice. Proc. Natl. Acad. Sci. U. S. A. 101:18111-18116.

16. Yang, S.H., Qiao, X., Fong, L.G., and Young, S.G. 2008. Treatment with a farnesyltransferase inhibitor improves survival in mice with a HutchinsonGilford progeria syndrome mutation. Biochim. Biophys. Acta. 1781:36-39.

17. Fong, L., et al. 2006. A protein farnesyltransferase inhibitor ameliorates disease in a mouse model of progeria. Science. 311:1621-1623.

18. Kieran, M.W., Gordon, L., and Kleinman, M. 2007. New approaches to progeria. Pediatrics. 120:834-841.

19. Gordon, L.B., et al. 2007. Disease progression in Hutchinson-Gilford progeria syndrome: impact on growth and development. Pediatrics. 120:824-833.

20. Toth, J.I., et al. 2005. Blocking protein farnesyltransferase improves nuclear shape in fibroblasts from humans with progeroid syndromes. Proc. Natl. Acad. Sci. U. S. A. 102:12873-12878.

21. Mallampalli, M.P., Huyer, G., Bendale, P., Gelb, M.H., and Michaelis, S. 2005. Inhibiting farnesylation reverses the nuclear morphology defect in a HeLa cell model for Hutchinson-Gilford progeria syndrome. Proc. Natl. Acad. Sci. U. S. A. 102:14416-14421.
22. Hirschman, J.E., and Jenness, D.D. 1999. Dual lipid modification of the yeast gy subunit Ste18p determines membrane localization of G $\beta \gamma$. Mol. Cell. Biol. 19:7705-7711.

23. Caplan, A.J., Tsai, J., Casey, P.J., and Douglas, M.G. 1992. Farnesylation of YDJ1p is required for function at elevated growth temperatures in Saccharomyces cerevisiae. J. Biol. Chem. 267:18890-18895.

24. Hancock, J.F., Magee, A.I., Childs, J.E., and Marshall, C.J. 1989. All ras proteins are polyisoprenylated but only some are palmitoylated. Cell. 57:1167-1177.

25. Troutman, J.M., Roberts, M.J., Andres, D.A., and Spielmann, H.P. 2005. Tools to analyze protein farnesylation in cells. Bioconjug. Chem. 16:1209-1217.

26. Dechat, T., et al. 2007. Alterations in mitosis and cell cycle progression caused by a mutant lamin A known to accelerate human aging. Proc. Natl. Acad. Sci.U.S. A. 104:4955-4960.

27. Coffinier, C., et al. 2008. A potent HIV protease inhibitor, darunavir, does not inhibit ZMPSTE24 or lead to an accumulation of farnesyl-prelamin A in cells. J. Biol. Chem. 283:9797-9804.

28. Yang, S.H., et al. 2008. Eliminating the synthesis of mature lamin reduces disease phenotypes in mice carrying a Hutchinson-Gilford progeria syndrome allele. J. Biol. Chem. 283:7094-7099.

29. Fong, L.G., et al. 2006. Prelamin A and lamin A appear to be dispensable in the nuclear lamina. J. Clin. Invest. 116:743-752.

30. Seabra, M.C., Ho, Y.K., and Anant, J.S. 1995. Deficient geranylgeranylation of Ram/Rab27 in choroideremia. J. Biol. Chem. 270:24420-24427.

31. Storch, S., Pohl, S., Quitsch, A., Falley, K., and Braulke, T. 2007. C-terminal prenylation of the CLN3 membrane glycoprotein is required for efficient endosomal sorting to lysosomes. Traffic. 8:431-444.

32. Moulson, C.L., et al. 2007. Increased progerin expression associated with unusual LMNA muta- 
tions causes severe progeroid syndromes. Hum. Mutat. 28:882-889.

33. Glynn, M.W., and Glover, T.W. 2005. Incomplete processing of mutant lamin A in Hutchinson-Gilford progeria leads to nuclear abnormalities, which are reversed by farnesyltransferase inhibition. Hum. Mol. Genet. 14:2959-2969.

34. Capell, B.C., et al. 2005. Inhibiting farnesylation of progerin prevents the characteristic nuclear blebbing of Hutchinson-Gilford progeria syndrome. Proc. Natl. Acad. Sci. U. S. A. 102:12879-12884.

35. Kirschner, J., et al. 2005. p.S143F mutation in lamin A/C: a new phenotype combining myopathy and progeria. Ann. Neurol. 57:148-151.

36. Verstraeten, V.L., et al. 2006. Compound heterozygosity for mutations in LMNA causes a progeria syndrome without prelamin A accumulation. Hum.
Mol. Genet. 15:2509-2522.

37. Fatkin, D., et al. 1999. Missense mutations in the rod domain of the lamin $\mathrm{A} / \mathrm{C}$ gene as causes of dilated cardiomyopathy and conduction-system disease. N. Engl. J. Med. 341:1715-1724.

38. Bonne, G., et al. 1999. Mutations in the gene encoding lamin $\mathrm{A} / \mathrm{C}$ cause autosomal dominant Emery-Dreifuss muscular dystrophy. Nat. Genet. 21:285-288.

39. Bonne, G., et al. 2000. Clinical and molecular genetic spectrum of autosomal dominant EmeryDreifuss muscular dystrophy due to mutations of the lamin A/C gene. Ann. Neurol. 48:170-180.

40. Mounkes, L.C., Kozlov, S., Hernandez, L., Sullivan, T., and Stewart, C.L. 2003. A progeroid syndrome in mice is caused by defects in A-type lamins. Nature. 423:298-301.
41. Arimura, T., et al. 2005. Mouse model carrying H222P-Lmna mutation develops muscular dystrophy and dilated cardiomyopathy similar to human striated muscle laminopathies. Hum. Mol. Genet. 14:155-169.

42. Mounkes, L.C., Kozlov, S.V., Rottman, J.N., and Stewart, C.L. 2005. Expression of an LMNA-N195K variant of A-type lamins results in cardiac conduction defects and death in mice. Hum. Mol. Genet. 14:2167-2180.

43. Scaffidi, P., and Misteli, T. 2006. Good news in the nuclear envelope: loss of lamin A might be a gain. J. Clin. Invest. 116:632-634.

44. Dalton, M., and Sinensky, M. 1995. Expression systems for nuclear lamin proteins: Farnesylation in assembly of nuclear lamina. Methods Enzymol. 250:134-148. 